A propos de risques et d'effets secondaires...

Je remercie Peppo Brandenberg pour son article qui illustre clairement les avantages, les risques et les défis du nombre minimal de cas. La question est si complexe qu'il est difficile d'y répondre au moyen d'un seul indicateur. De plus, les effets secondaires du risque d'une augmentation du volume des prestations doivent impérativement être contenus au moyen d'un élément assurant la qualité de l'indication telles que les mesures des résultats de santé rapportés par les patients (PROM).

\title{
Nombre minimal de cas - gage de qualité ou succédané?
}

\author{
Josef E. Brandenberg
}

Dr méd., président de la FMCH

Les références se trouvent sous www.bullmed.ch $\rightarrow$ Numéro actuel ou $\rightarrow$ Archives $\rightarrow 2018 \rightarrow 48$.
Sérieusement, chers collègues, seriez-vous d'accord de vous faire opérer par un chirurgien qui ne réalise que rarement l'intervention que vous devrez subir? Je suppose que non. Et en regard de vos connaissances, vous ne seriez certainement pas non plus disposés à subir une intervention chirurgicale dans un hôpital qui cumule les mauvais résultats, même si le nombre de cas y est très élevé.

Selon une enquête représentative sur mandat de l'assureur automobile allemand «DA direkt», c'est la pratique qui fait un conducteur [1]. Dans le sport d'élite, le succès est le fruit de nombreuses années d'entraînement et les grands musiciens passent leur vie à exercer leur voix ou leur instrument plusieurs heures par jour. Cela s'applique également à la médecine et en particulier aux disciplines chirurgicales. En 2002, Halm et al. [2] ont évalué 272 publications scientifiques dans une métaanalyse: $71 \%$ d'entre elles établissent un lien entre le nombre plus élevé de cas dans un hôpital et les bons résultats de santé. Pour $69 \%$ des études, les résultats sont meilleurs lorsque le nombre d'interventions par chirurgien est plus élevé. Ce constat est le plus significatif pour les anévrismes aortiques, les tumeurs du pancréas et celles de l'œsophage [3], ce que confirme une récente étude qui s'applique également à la Suisse. Dans ce contexte, personne ne contestera qu'il est pertinent que la Conférence suisse des directrices et directeurs cantonaux de la santé (CDS) n'attribue, depuis 2009, des mandats de prestations pour divers traitements hautement spécialisés qu'à des établissements figurant sur les listes hospitalières et atteignant les nombres minimaux de cas requis par an et par traitement/intervention.

Cette prescription d'un nombre minimal de cas par établissement contribue-t-elle à assurer la qualité? En partie seulement.
Les conditions infrastructurelles, techniques et organisationnelles sont certes indispensables pour le traitement des brûlés graves ou pour la transplantation d'organes mais c'est l'expérience du chirurgien qui reste déterminante pour le succès de l'intervention. Dans un hôpital, la répartition des opérations rares sur plusieurs chirurgiens est préjudiciable à la qualité. Introduire un nombre minimal de cas pour des interventions rares et hautement spécialisées, d'accord, mais alors au moins par chirurgien et non par hôpital.

Comme il l'avait déjà fait en 2011 pour les hôpitaux, le canton de Zurich a introduit au $1^{\mathrm{er}}$ janvier 2018 un nombre minimal de cas pour les chirurgien(ne)s («Ergänzende Anforderungen zur Qualitätssicherung» en allemand). Ces exigences ne s'appliqueront désormais plus seulement aux interventions chirurgicales hautement spécialisées, mais également aux interventions courantes [4] et concerneront six domaines de prestations hospitalières en gynécologie, urologie et chirurgie de l'appareil locomoteur.

Les nombres minimaux de cas améliorent-ils la qualité? Là aussi, en partie seulement.

Il s'est avéré que dans le domaine des endoprothèses, un faible nombre de cas entraîne un taux plus élevé d'opérations de révision comme le montre l'étude de Manley et al. [5] également représentative d'autres publications scientifiques. Différentes études indiquent cependant que la qualité n'est pas ou pas uniquement corrélée au nombre de cas par hôpital ou par praticien, mais surtout à l'âge de celui-ci [6], à sa formation [7] et à sa spécialisation [8]. Ces éléments sont d'ailleurs pris en compte depuis de nombreuses années dans la formation aux disciplines chirurgicales, qui exige de remplir un catalogue des opérations pour obtenir le titre de spécialiste. 
Les pilotes de ligne aussi doivent avoir un certain nombre d'heures de vol à leur actif et accomplir un nombre minimum de vols par an après l'obtention de leur licence. Il est donc normal d'exiger des spécialistes un nombre minimal d'opérations au terme de leur formation postgraduée. A l'instar de l'aviation, d'autres facteurs que l'expérience entrent également en ligne de compte comme ce fut le cas lors de deux accidents tragiques récents où les trois pilotes étaient hautement qualifiés et expérimentés.

Sur la base d'une étude de longue durée qui s'est déroulée de 1990 à 2004, Kurtz et al. [9] montrent que les opérations de révision augmentent avec l'âge du chirurgien, même si ce dernier excelle dans son domaine et qu'il a accompli un grand nombre d'opérations. Un fait qui ne dépend pas d'une baisse de qualité, mais de l'usure du matériel implanté. Chez les chirurgiens aussi, le temps laisse des traces, comme l'évoque le Prof. Hardy Weber, ancien médecin-chef du service d'orthopédie de l'Hôpital cantonal de Saint-Gall.

Le registre australien des implants [10] illustre également l'impact de l'implant sur les taux de révision. Les «High volume surgeons» qui effectuent plus de 70 opérations du genou par an font face à moins de complications à court terme, mais génèrent des taux de révision plus élevés à moyen et à long terme par la pose d'implants de mauvaise qualité. Le récent exemple du resurfaçage des hanches (implants de resurfaçage) montre également qu'en Suisse les chirurgiens orthopédiques qui ont posé un grand nombre de ces implants ont occasionné plus de dommages que ceux qui ont effectué moins d'opérations, mais utilisé des implants standards ayant fait leurs preuves. Mieux vaut donc réaliser moins d'opérations, mais de bonne qualité.

Selon quels critères les nombres minimaux de cas doivent-ils être fixés? Ou plutôt selon quels critères les nombres minimaux de cas permettent-ils d'obtenir de meilleurs résultats?

Une étude de Schräder et al. [11] souligne également la difficulté à définir des valeurs limites. A l'instar du sport d'élite ou des arts, il est nécessaire de s'exercer en permanence pour réussir, mais sans talent, même l'entraînement le plus poussé sera vain. On trouve aussi des chirurgiens qui feraient mieux de s'abstenir d'opérer. C'est un peu pareil pour les automobilistes qui, selon l'étude susmentionnée [1], sont 9 sur 10 à s'estimer très bon conducteur.

L'étude précitée sur le groupe de chirurgiens de Philadelphie [9] qualifie les opérateurs de «Top of Surgeons TOS» s'ils ont implanté plus de 25 à 45 prothèses de hanche et plus de 33 à 86 prothèses de genou. Aux Etats-Unis, seuls $5 \%$ des spécialistes en orthopédie seraient en mesure d'atteindre un tel nombre d'interventions, ce qui illustre les défaillances du système. Dans le canton de Zurich à l'avenir, tout chirurgien qui n'atteindra que le nombre minimal requis de 15 prothèses de hanche et de 15 prothèses du genou par an ne comptera pas parmi les «Top of Surgeons».

Swiss Orthopaedics a souvent souligné un autre problème lié au nombre minimal de cas [12,13]: l'augmentation du nombre de prestations pour atteindre les valeurs limites fixées. En exigeant une répartition des points pour attester les nombres de cas minimaux, la direction de la santé du canton de Zurich encourage indirectement l'augmentation du nombre de prestations étant donné que, paradoxalement, les points attribués sont divisés par deux si deux spécialistes opèrent ensemble. Par ailleurs, cela torpille l'objectif d'améliorer la qualité. Or l'expérience montre que quatre yeux valent mieux que deux, que la durée de l'intervention est plus courte et que l'indication est vérifiée mutuellement. Compte tenu du rôle précurseur du canton de Zurich et de l'aval de la plus haute instance juridique [14] du pays, les nombres minimaux de cas deviendront bientôt une réalité dans toute la Suisse et pourraient aussi être étendus aux interventions chirurgicales ambulatoires.

En conclusion, fixer des nombres minimaux de cas sans prendre en compte la qualité de l'indication, l'expérience du médecin, le choix de l'implant, sans appréciation des résultats de santé et avec des valeurslimites aléatoires revient à recourir à un succédané sans réelle amélioration de la qualité. 